\title{
Dynamic Cell and Microparticle Control via Optoelectronic Tweezers
}

\author{
Aaron Takami Ohta, Student Member, IEEE, Pei-Yu Chiou, Tae H. Han, James C. Liao, Urvashi Bhardwaj, \\ Edward R. B. McCabe, Fuqu Yu, Ren Sun, and Ming C. Wu, Fellow, IEEE
}

\begin{abstract}
This paper reports on cell and microparticle manipulation using optically induced dielectrophoresis. Our novel optoelectronic tweezers (OET) device enables optically controlled trapping, transportation, and sorting via dielectrophoretic forces. By integrating a spatial light modulator and using direct imaging, arbitrary dynamic manipulation patterns are obtained. Here, we demonstrate manipulation functions, including particle collectors, single-particle traps, individually addressable single-particle arrays, light-defined particle channels, and size-based particle sorting. OET-induced particle manipulation velocities are analyzed as a function of the applied voltage, optical pattern linewidth, and single-particle trap dimensions.

[2006-0210]
\end{abstract}

Index Terms-Dielectrophoresis (DEP), optical tweezers, optically induced DEP, optoelectronic tweezers (OET).

\section{INTRODUCTION}

$\mathbf{M}$ ICROSCALE manipulation is an important tool in many fields, including biological research and colloidal science. Dynamic easily reconfigurable micromanipulation tools are highly sought.

One mature cellular-scale manipulation technology is optical tweezers, which provides a noninvasive method of manipulating particles with sizes from approximately $100 \mu \mathrm{m}$ down to a few nanometers [1]. Holographic techniques have been used to increase the functionality of optical tweezers to include particle sorting [2], 3-D manipulation [3], and the creation of multiple optical traps [4], [5]. However, optical tweezers are limited by high optical power requirements. A typical trap requires at least $1 \mathrm{~mW}$ of laser power, whereas the multiple traps created by holographic optical tweezers demand source powers of $500 \mathrm{~mW}$ to $1 \mathrm{~W}$ [5], [6]. Such high power levels, especially in the visible wavelengths, can result in optical and/or thermal damage to live biological specimens [7]-[12]. Furthermore, as optical tweezers relies on the gradient force of the optical beam to trap particles, the laser light must be highly focused. Typically, high-numerical-aperture lenses are employed to achieve optical trapping, limiting the manipulation area of most optical tweezers to a field of view of less than $100 \mu \mathrm{m}$. This represents

Manuscript received September 26, 2006; revised February 28, 2007. This work was supported in part by the Center for Cell Mimetic Space Exploration, a NASA University Research, Engineering and Technology Institute, under Award NCC 2-1364. Subject Editor H. Zappe.

A. T. Ohta and M. C. Wu are with the University of California, Berkeley, CA 94720 USA (e-mail: aohta@eecs.berkeley.edu; wu@eecs.berkeley.edu).

P.-Y. Chiou, T. H. Han, J. C. Liao, U. Bhardwaj, E. R. B. McCabe, F. Yu, and R. Sun are with the University of California, Los Angeles, CA 90095 USA (e-mail: pychiou@ seas.ucla.edu).

Digital Object Identifier 10.1109/JMEMS.2007.896717 a bottleneck in the parallel processing of biological cells (which may have diameters of $10 \mu \mathrm{m}$ or greater) and larger particles. Optical tweezers are also limited to attractive forces for most biological particles such as cells.

Dielectrophoresis (DEP) is another widely used microparticle manipulation technique [13]. It has been demonstrated on particles with sizes ranging from approximately $1 \mathrm{~mm}$ down to $14 \mathrm{~nm}$ [14]. Unlike optical tweezers, DEP is capable of a large effective particle manipulation area, is limited only by the size of the device, and can produce repulsive forces in addition to attractive forces. However, a limitation of conventional DEP is the static pattern of electrodes used for particle manipulation, reducing the flexibility of this technology.

We have demonstrated another method for the optical manipulation of micrometer-scale objects: optically induced DEP or optoelectronic tweezers (OET) [15]. Using a laser to induce dielectrophoretic forces, we have demonstrated controlled movement of $25-\mu \mathrm{m}$ polystyrene particles and $E$. coli bacteria [16], [17]. This technique allows the use of very low optical power levels, enabling particle manipulation with an incoherent light source. The integration of a spatial light modulator into the optical system enables dynamic reconfigurable optical patterns, providing increased functionality and versatility in particle manipulation. Using incoherent-light-actuated OET, we have demonstrated the creation of 15000 particle traps over an area of $1.3 \mathrm{~mm}^{2}$, size-based particle sorting, and the selective concentration of live and dead human B cells [15]. In addition, another group has used optically induced DEP to form virtual channels and to trap yeast cells [18]-[20].

In this paper, we present the dynamic direct-image-driven manipulation of live red and white blood cells and microparticles using incoherent-light-driven OET. We demonstrate many particle manipulation techniques, including particle collectors, single-particle traps, individually addressable single-particle arrays, light-defined particle channels, and size-based particle sorting. Critical parameters for the optimization of OET manipulation patterns are analyzed, including the dependence of the OET-induced manipulation velocity on applied voltage, optical pattern linewidth, and single-particle trap dimensions.

\section{DEP}

The actuation forces of OET are identical to those of DEP, which refers to the forces induced upon a particle in the presence of a nonuniform electric field [13]. A particle within the electric field forms an induced dipole, which experiences 


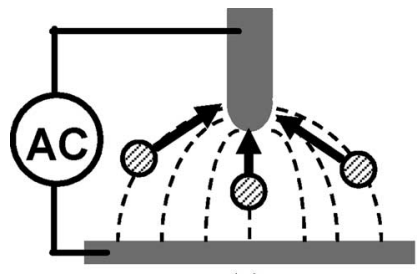

(a)

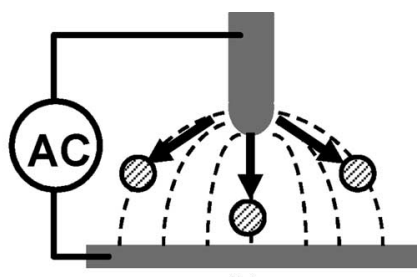

(b)
Fig. 1. Frequency dependence of dielectrophoretic force. (a) Positive DEP, in which particles are attracted toward electric field maxima, occurs when $\operatorname{Re}[K(\omega)]>0$. (b) Negative DEP, in which particles are repelled from field maxima, occurs when $\operatorname{Re}[K(\omega)]<0$.

a force dependent upon the field gradient (for a spherical particle), i.e.,

$$
F_{\mathrm{DEP}}=2 \pi r^{3} \varepsilon_{m} \operatorname{Re}[K(\omega)] \nabla\left(E^{2}\right)
$$

where $r$ is the particle radius, $\varepsilon_{m}$ is the permittivity of the media surrounding the particle, $E$ is the root-mean-square value of the electric field, and $\operatorname{Re}[K(\omega)]$ is the real part of the Clausius-Mossotti factor, i.e.,

$$
K(\omega)=\frac{\varepsilon_{p}^{*}-\varepsilon_{m}^{*}}{\varepsilon_{p}^{*}+2 \varepsilon_{m}^{*}}, \quad \varepsilon_{p}^{*}=\varepsilon_{p}-j \frac{\sigma_{p}}{\omega} ; \quad \varepsilon_{m}^{*}=\varepsilon_{m}-j \frac{\sigma_{m}}{\omega}
$$

where $\sigma$ is the conductivity of the particle or the medium, and $\omega$ is the angular frequency of the electric field. The magnitude of $\operatorname{Re}[K(\omega)]$ varies with frequency, resulting in frequency dependence of the dielectrophoretic force (Fig. 1). Positive values of $\operatorname{Re}[K(\omega)]$ result in particle attraction to electric field maxima (positive DEP). For negative values of $\operatorname{Re}[K(\omega)]$, particles are repelled from field maxima (negative DEP). Applying an ac electric field thus allows the tuning of the type of DEP force induced on a particle, as well as negating any electrophoretic effects, or particle movement due to its surface charge.

In conventional DEP, the nonuniform electric fields are generated by a variety of static electrode configurations [21]-[23]. To create dynamic nonuniform fields for reconfigurable DEP traps, complex control circuitry using CMOS technology is required [24]. In contrast, optoelectronics tweezers enable lightpatterned virtual electrodes to address dielectrophoretic forces. It is flexible and reconfigurable and can continuously address DEP forces across a 2-D surface.

\section{OET}

\section{A. Device Structure and Fabrication}

The lower photoconductive surface of the OET device consists of an indium-tin-oxide (ITO)-coated glass substrate, a 10-nm aluminum film to reduce contact resistance, a $1-\mu \mathrm{m}$ undoped amorphous silicon (a-Si) photoconductive layer, and a 20 -nm silicon nitride passivation layer. A liquid solution containing the particles of interest is sandwiched between the photoconductive surface and an upper ITO-coated glass slide (Fig. 2). A 100- $\mu \mathrm{m}$ spacer is used to maintain the gap between the upper and lower surfaces. An ac bias across the top and bottom ITO layers is applied to power the device.

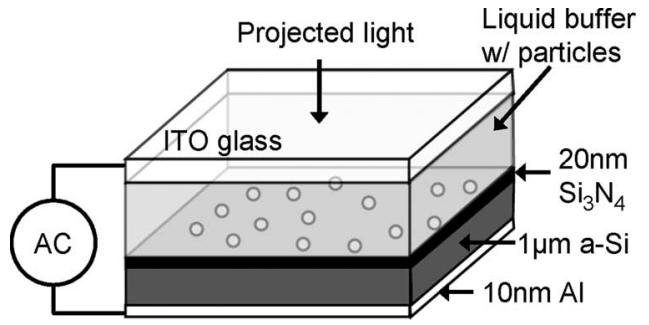

Fig. 2. OET device structure. A liquid solution containing the particles of interest is contained between an upper electrode surface (ITO glass) and a lower photoconductive layer (a-Si), separated by a $100-\mu \mathrm{m}$ spacer (not shown).

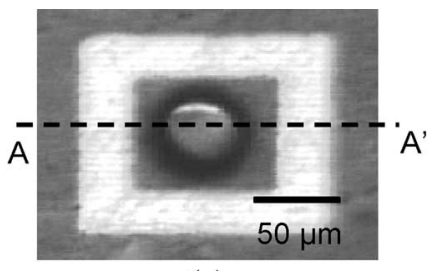

(a)

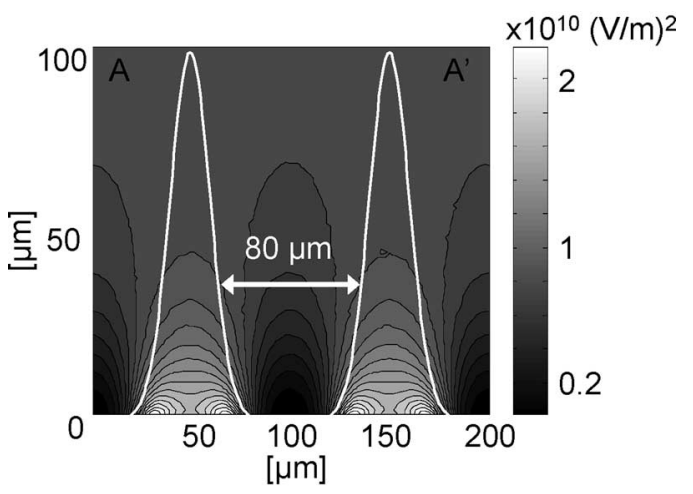

(b)

Fig. 3. (a) Single-particle OET trap. A $45-\mu$ m polystyrene sphere is contained by optically induced negative DEP. (b) Distribution of the square of the electric field for the single-particle trap along the cross section A-A'. The DEP force is proportional to the gradient of this distribution. The profile of the projected $20-\mu \mathrm{m}$-wide light pattern, which is approximated by Gaussian beams, is represented by the white lines.

\section{B. Optically Induced DEP}

The OET device enables optically induced DEP by exploiting the photoconductive properties of the amorphous silicon layer. Amorphous silicon has a very high electrical impedance in the dark; thus, most of the applied voltage drops across the a-Si layer, resulting in a low electric field in the liquid layer. However, focusing incident light onto the a-Si surface creates electron-hole pairs, reducing the impedance of a-Si layer by several orders of magnitude. As a result, a "virtual electrode" is created in the illuminated area. This switches the majority of the voltage drop to the liquid layer, forming a high electric field in the liquid above the virtual electrode. The resulting electric field gradient in the liquid layer creates dielectrophoretic force for microparticle manipulation.

To illustrate, a repulsive OET trap is shown in Fig. 3(a). This single-particle rectangular trap has inner dimensions of $80 \times 60 \mu \mathrm{m}$. A sphere with a diameter of $45 \mu \mathrm{m}$ is surrounded by light "walls" $20 \mu \mathrm{m}$ in width. The corresponding crosssectional distribution of the square of the electric field was 
TABLE I

Multiphysics Simulation PARAmeters

\begin{tabular}{ll}
\hline \hline Parameter & Value \\
\hline a-Si peak conductivity & $100 \mathrm{mS} / \mathrm{m}$ \\
a-Si relative permittivity & 11 \\
Liquid conductivity & $10 \mathrm{mS} / \mathrm{m}$ \\
Liquid relative permittivity & 80 \\
Applied voltage & $10 \mathrm{~V}$ \\
FWHM beam width & $20 \mu \mathrm{m}$ \\
\hline \hline
\end{tabular}

simulated using a commercial finite-element modeling program (FEMLAB, now COMSOL Multiphysics [25]). The simulation parameters are based on empirically determined material properties and are given in Table I. The conductivity of the a-Si is assumed to be proportional to the illuminated light intensity, with a peak value of $100 \mathrm{mS} / \mathrm{m}$. The intensity of the illumination patterns is assumed to have a Gaussian profile. The simulation result shows that the width of the trap as experienced by the particle is approximately $50 \mu \mathrm{m}$, as DEP force depends on the gradient of this distribution [Fig. 3(b)]. If negative DEP forces are induced by the trap pattern, all particles outside the trap area will be repelled by the electric field maxima forming the trap perimeter. Any particle within the enclosed trap area will feel similar repulsive forces, but these forces balance, trapping the particle. Once a particle is contained within the rectangular pattern, the trap can be moved, transporting the particle to a desired location. Furthermore, multiple traps can be used as building blocks to form arrays of trapped particles. Such arrays can be arbitrarily arranged and dynamically reconfigured.

\section{Experimental Setup}

Polystyrene beads of various sizes (5-, 10-, 20-, 25-, or $45-\mu \mathrm{m}$ diameters, from Polysciences, Inc.) are suspended in a liquid solution consisting of deionized water and $\mathrm{KCl}$ salt, which are mixed to obtain a conductivity of $10 \mathrm{mS} / \mathrm{m}$. Live bovine red blood cells or live human B cells are suspended in an isotonic solution of $8.5 \%$ sucrose and $0.3 \%$ dextrose. The suspended cells or microparticles are introduced into the OET device, which is placed on a microscope for observation. A charge-coupled device (CCD) camera that is attached to the observation port of the microscope records images and video of the experiments. To produce the electric field that is necessary for DEP, an ac voltage (Agilent 33120A) is applied across the top ITO surface and the bottom photoconductive surface of the OET device.

The light source in some prior OET experiments consisted of a $0.8-\mathrm{mW} \mathrm{He}-\mathrm{Ne}$ laser [16], [17]. However, the low optical power requirement of OET enables the use of an inexpensive incoherent light source such as a mercury or halogen lamp or a LED (Fig. 4).

In addition, we can produce light patterns by imaging technique rather than scanning technique. With no need to focus all optical energy, we can use a spatial light modulator to directly pattern images rather than the holographic techniques that are employed by optical tweezers arrays [4]-[6]. Our current setup uses a $1024 \times 768$ pixel digital micromirror device (DMD)

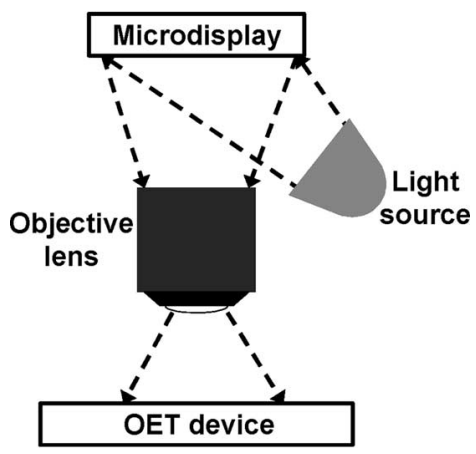

Fig. 4. Experimental setup for OET. The light source is incoherent (mercury or halogen lamp or LED).

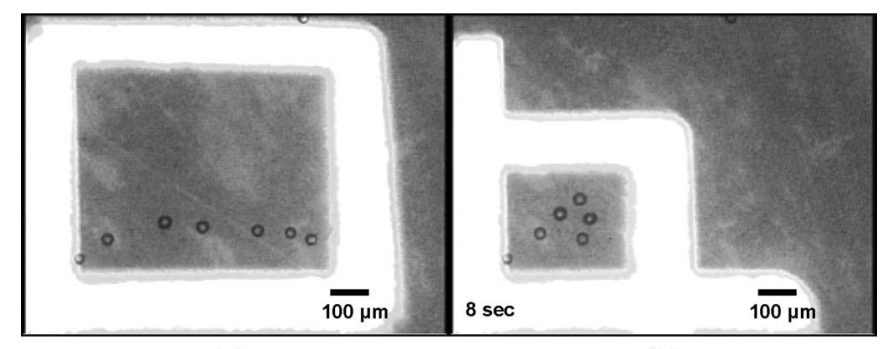

(a)

(b)

Fig. 5. Collection of $25-\mu \mathrm{m}$-diameter polystyrene beads in a solution via negative DEP. The incoherent light patterns are generated with a micromirror array and projected onto the OET device.

from Texas Instruments. Each pixel of the DMD is $13.68 \times$ $13.68 \mu \mathrm{m}$. The image from the DMD is focused using a $10 \times$ objective lens $(\mathrm{NA}=0.30)$ and projected onto the OET device. Control of the projected images is accomplished via a PC interface to the DMD.

\section{EXPERIMENTAL RESUlts}

\section{A. Microparticle Manipulation}

One type of optical setup for OET manipulation using an incoherent light source is a DMD-based digital projector (InFocus LP335). The projector displays images that are drawn on a PC in Microsoft PowerPoint software. In this setup, the projector provides both the optical source (a high-pressure mercury lamp) and the DMD-to-PC interface. The output of the projector is collected, collimated, and directed into the $10 \times$ objective lens, projecting an image onto the OET device. The power at the projector output was measured to be approximately $600 \mathrm{~mW}$. Approximately $7 \%$ of this power is collected by the objective lens and focused onto the OET device. Therefore, the power of the light incident on the OET surface (not including losses through the bulk optics) is $42 \mathrm{~mW}$, corresponding to an intensity of $120 \mathrm{nW} / \mu \mathrm{m}^{2}$.

Negative DEP forces were employed to collect randomly distributed particles (Fig. 5). As the light pattern sweeps across the manipulation area, particles are pushed into the desired location.

Using different image patterns, we were able to create dynamic single-particle ring traps containing a single $25-\mu \mathrm{m}$ 


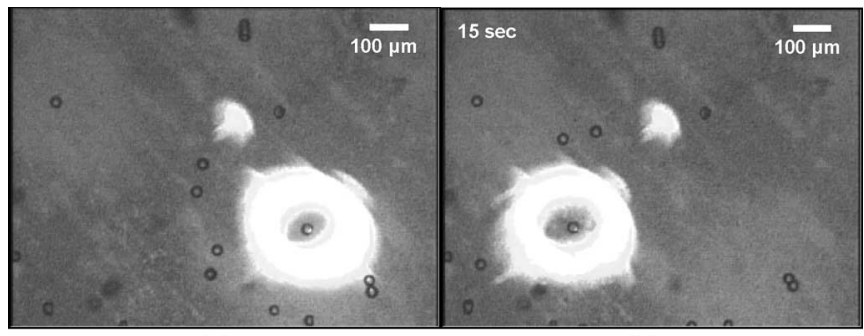

(a)

(b)

Fig. 6. (a) Single-particle trap is formed using negative DEP. (b) As the light pattern is moved, a single $25-\mu \mathrm{m}$-diameter polystyrene bead remains in the trap, whereas the beads outside the trap are repelled.

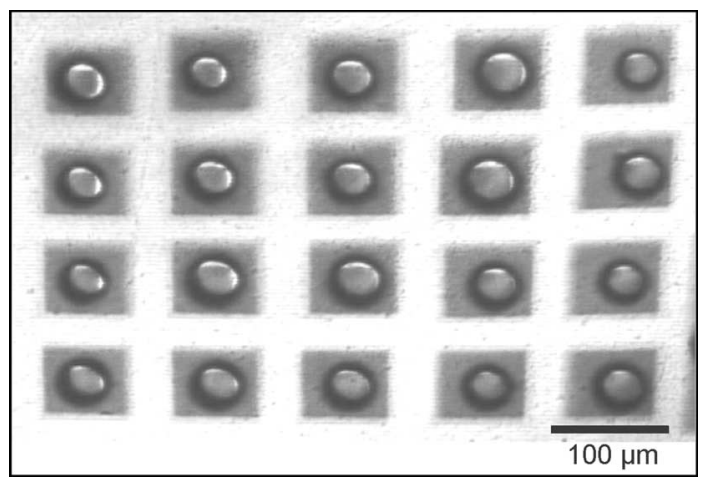

Fig. 7. Array of single $45-\mu$ m-diameter beads formed from multiple singleparticle square traps. Each particle is individually addressable. The time required to form this array of 20 particles was $3 \mathrm{~min}$.

diameter polystyrene bead via negative DEP (Fig. 6). These traps were translated in real time under user control, at velocities of up to $40 \mu \mathrm{m} / \mathrm{s}$.

Using multiple instances of these single-particle traps, individually addressable arrays of particles can be formed. Each randomly positioned particle is first contained within a square trap, which is a procedure achieved in real time by manual user control of the image pattern. The multiple traps can then be positioned to form an array of individually addressable cells. Using this technique, we were able to form a $5 \times 4$ array of single-particle traps (Fig. 7). We were able to move the resulting array of single particles around the image plane at approximately $25 \mu \mathrm{m} / \mathrm{s}$. Although the formation of the array was performed manually, it can be automated by combining OET with an image-analysis feedback-control system, as demonstrated in [26] and [27]. Biological applications of such an array include studies on single-cell behavior and interactions.

Since each cell of the array is an independent single-particle trap, the array has the capability of being dynamically rearranged. To illustrate, an array consisting of 45- and $20-\mu \mathrm{m}$ polystyrene beads is reorganized under operator control (Fig. 8). This demonstrates the addressability of each particle trap, as well as the dynamic nature of the OET patterns.

By adjusting the projected image, other particle manipulation schemes are achievable. Negative DEP can be used to guide particles along light-defined "channels" at velocities of approximately $10 \mu \mathrm{m} / \mathrm{s}$ (Fig. 9). In addition, due to the ease of reconfiguration of the manipulation pattern, we can implement

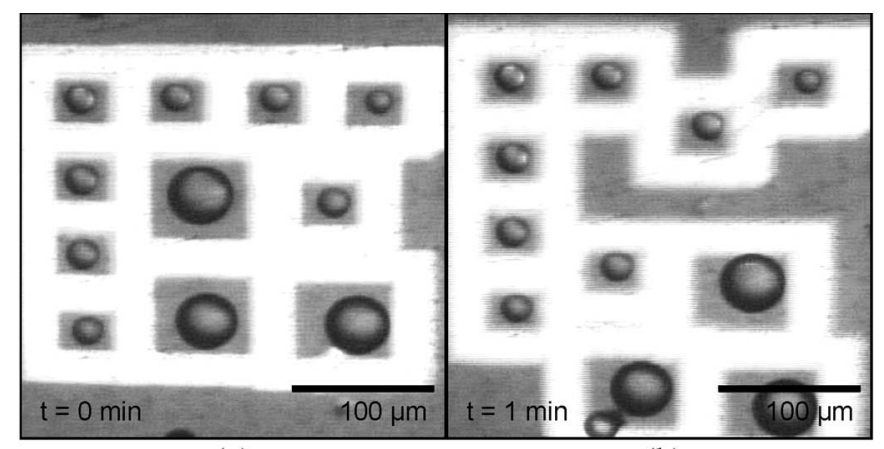

(a)

(b)

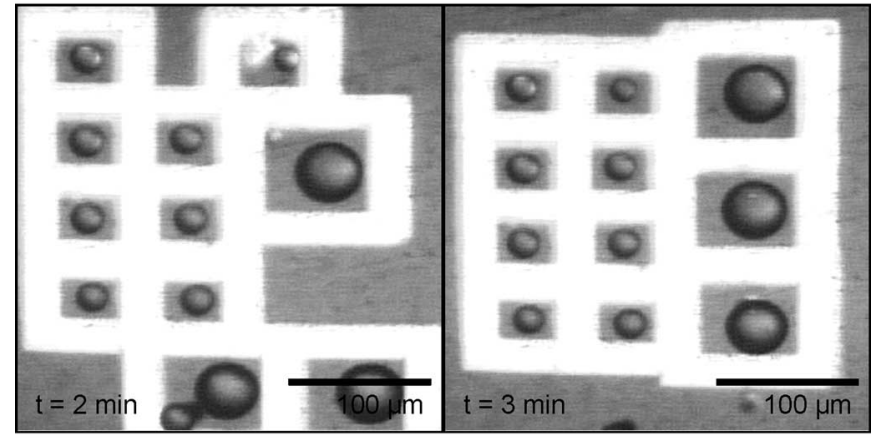

(c)

(d)

Fig. 8. Dynamic rearrangement of an array containing both $45-$ and $20-\mu \mathrm{m}$ particles. An array is rearranged by moving individual cells into a desired configuration. Total rearrangement time is $3 \mathrm{~min}$.

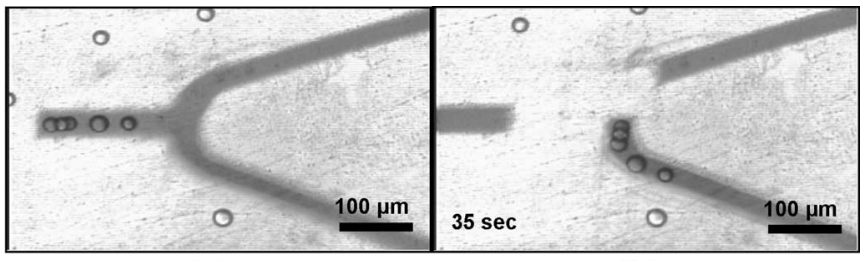

(a)

(b)

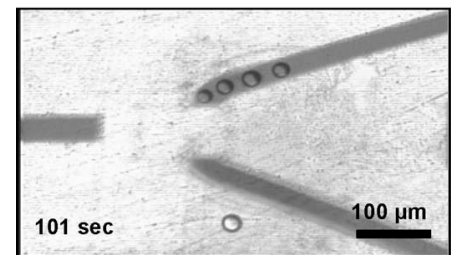

(c)

Fig. 9. (a) Polystyrene beads in a light-defined channel. The 20- $\mu \mathrm{m}$ particles can be directed down either the (b) lower branch or (c) upper branch under direct user control.

user-controlled "valves" in the channels to allow selective particle transport between channels [Fig. 9(b) and (c)].

Other patterns can be utilized for sorting particles based on size. Here, we use a "comb" configuration, which utilizes a periodic alternating illuminated/nonilluminated line (Fig. 10). This comb is swept across the manipulation area at a rate of $25 \mu \mathrm{m} / \mathrm{s}$. Smaller (20- $\mu \mathrm{m}$-diameter) beads are able to escape through the gaps in the electric field potential between the illuminated areas. However, these gaps are too small to allow the larger (45- $\mu \mathrm{m}$-diameter) beads to escape. Thus, the smaller beads are left behind, whereas the larger beads are swept out of the manipulation area. 


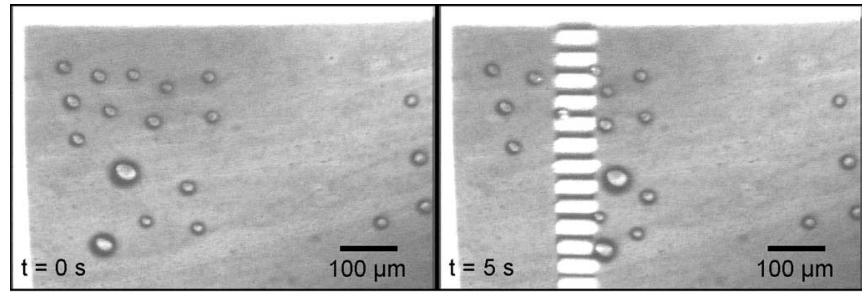

(a)

(b)

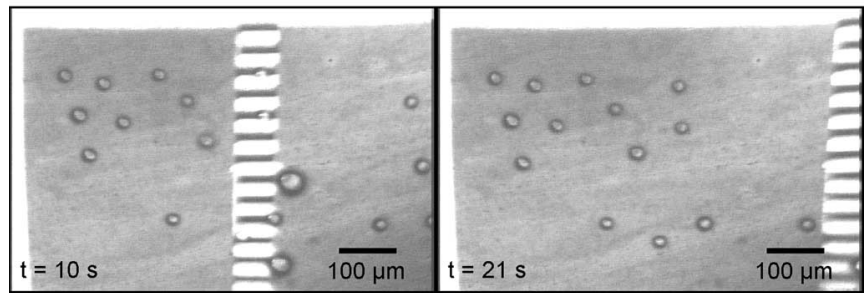

(c)

(d)

Fig. 10. Sized-based sorting of $20-$ and $45-\mu \mathrm{m}$-diameter beads. (b)-(d) "Comb" pattern is swept across the manipulation area. (b) and (c) Larger $45-\mu \mathrm{m}$ beads are pushed to the right. (d) Smaller 20- $\mu \mathrm{m}$ beads escape through the dark gaps in the light pattern and remain in the manipulation area.

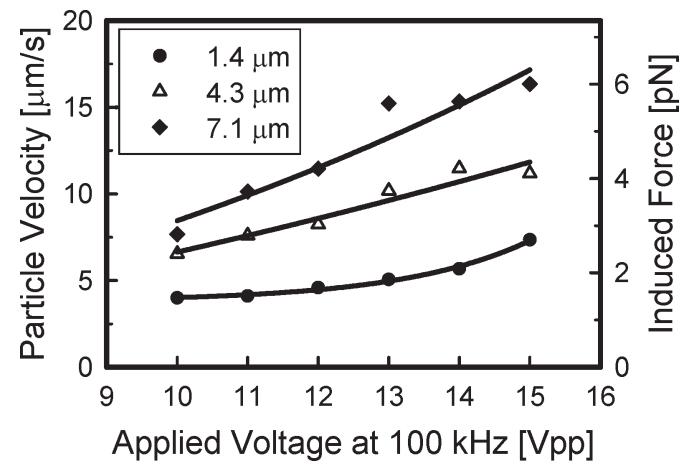

Fig. 11. Induced particle velocity (measured) and force (calculated) as a function of applied voltage at $100 \mathrm{kHz}$ for several illumination pattern widths. Mean particle diameter is $20 \mu \mathrm{m}$.

Induced particle velocities were measured using a LED source (Luxeon Star/O, $\lambda=625 \mathrm{~nm}$ ), which provided a power density of $10.8 \mathrm{nW} / \mu \mathrm{m}^{2}$. The maximum manipulation velocity of $20-\mu \mathrm{m}$-diameter beads is a function of several factors. As expected, the velocity increases as the applied voltage is increased (Fig. 11). In steady state, the OET force is balanced by the viscous drag of the fluid, which is related to the velocity of a spherical particle by Stokes' law, i.e., $F=6 \pi r \eta v$, where $r$ is the radius of the particle, $\eta$ is the viscosity of the fluid, and $v$ is the velocity of the particle. We can therefore use Stokes' law to calculate the OET force on a particle.

Increasing the applied voltage translates into a sharper electric field gradient, increasing the DEP force [see (1)]. However, DEP forces are typically proportional to the square of the applied voltage; our results show a more linear relationship. We have also found that particle velocity is dependent on the linewidth of the manipulation pattern, which is a phenomenon that is unique to our manipulation technique (Fig. 12). The trends observed in Figs. 11 and 12 can be attributed to the tendency of polystyrene particles to levitate vertically, due to the vertical gradient of the electric field [Fig. 3(b)]. As a

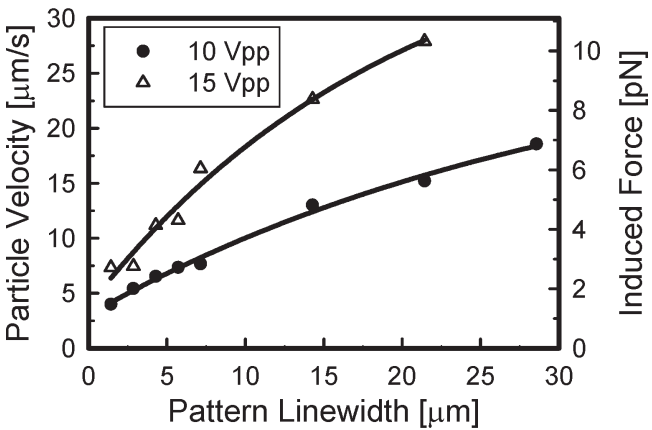

Fig. 12. Induced particle velocity (measured) and force (calculated) as a function of the illuminated linewidth of the manipulation pattern. The lower curve is for an applied bias of $10 \mathrm{Vpp}$ at $100 \mathrm{kHz}$; the top curve is for a bias of $15 \mathrm{Vpp}$ at $100 \mathrm{kHz}$. Mean particle diameter is $20 \mu \mathrm{m}$

result, as the optical patterns are swept across the manipulation area, particles move in both the lateral and vertical directions. Furthermore, as the applied voltage is increased, both the lateral and vertical gradients increase proportionally. However, in the case of these experiments, an increase in the vertical gradient is undesirable. The increased vertical gradient will result in increased particle levitation, which reduces the lateral force induced on a particle. As a particle is levitated away from the a-Si OET surface, the lateral field gradient decreases [Fig. 3(b)], resulting in reduced lateral force. Similarly, a pattern with a narrow linewidth cannot be moved very rapidly before a particle's tendency to levitate causes it to rise over the potential "wall" created by the pattern's corresponding electric field. However, this problem is alleviated by utilizing wider linewidth patterns. It may also be possible to reduce this effect by using a smaller spacing between the top and bottom OET surfaces.

Another factor affecting single-particle manipulation velocities is the dimensions of the single-particle trap [Fig. 13(a)]. The inner trap width is the measured distance between the light patterns forming the "walls" of the trap. These data agree with simulations [Fig. 3(b)] that show that the electric field gradient extends beyond the illuminated optical pattern. This results in the levitation of particles in traps with smaller inner dimensions. This levitation, in turn, causes a reduction in the lateral induced force and makes the single-particle trap more unstable. Measurements performed with inner trap widths of less than $35 \mu \mathrm{m}$ showed that these traps are too unstable to provide reliable particle movement in the desired direction. The corresponding simulations show [Fig. 3(b)] that for the smaller trap dimensions, the center of the trap is a local minimum. However, as the trap is moved, particles can easily migrate to the outside of the trap, which has a lower potential.

\section{B. Mammalian Cell Manipulation}

The results achieved using polystyrene beads are not exactly representative of live mammalian cells. The beads used in the OET experiments are homogeneously composed of polystyrene, whereas mammalian cells have a more complex structure consisting of a phospholipid membrane, cytoplasm, and multiple organelles. To model DEP force on cells, a singleshell representation is used [Fig. 14(a)] [28]. The permittivity and conductivity of the cell membrane and interior are used to 


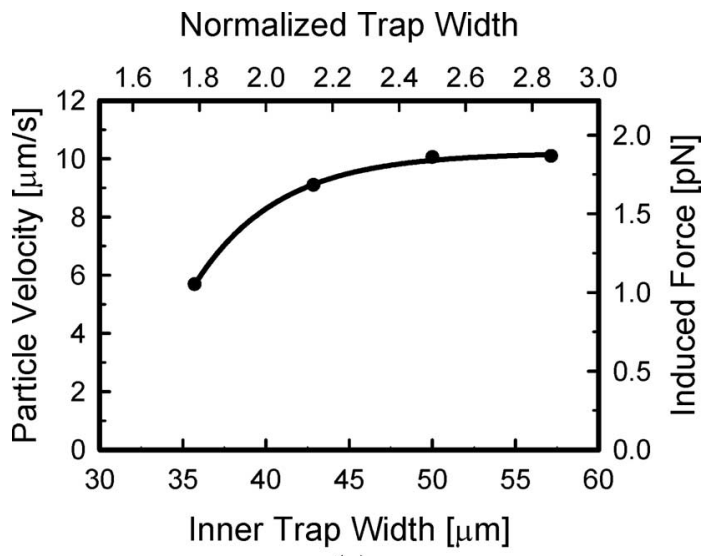

(a)

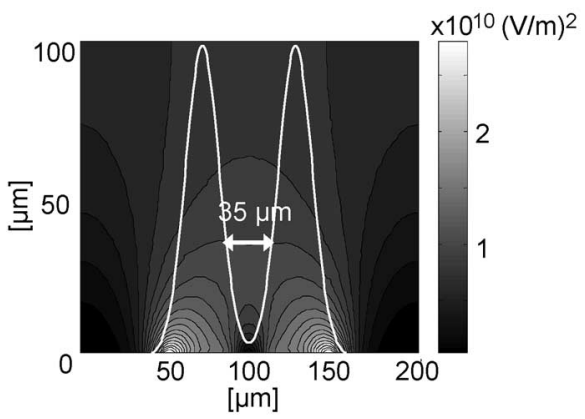

(b)

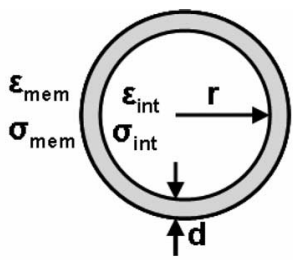

(a)

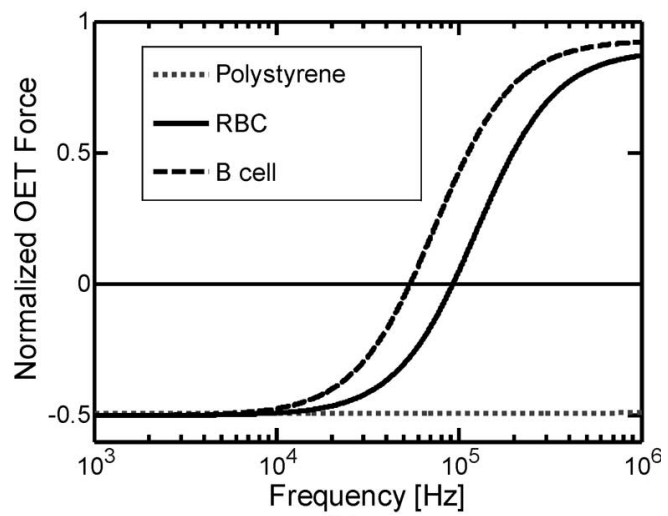

(b)

Fig. 14. (a) Single-shell model of a typical mammalian cell. (b) Normalized DEP force for three different types of microparticles in a $10-\mathrm{mS} / \mathrm{m}$ aqueous solution as a function of the frequency of the applied electric field. Positive values indicate an attractive force, whereas negative values indicate a repulsive force.

TABLE II

Fig. 13. (a) Induced particle velocity (measured) and force (calculated) as a function of inner trap dimensions that were measured between the light patterns forming the trap walls. Mean particle diameter is $20 \mu \mathrm{m}$. Applied bias is $10 \mathrm{Vpp}$ at $100 \mathrm{kHz}$. (b) Simulated square of the electric field for a $35-\mu \mathrm{m}-$ inner-diameter particle trap.

determine an effective complex permittivity that is used in (2). This effective permittivity is given by [28]

$$
\varepsilon_{p}^{*}=C_{\mathrm{mem}}^{*} \frac{3 r \varepsilon_{\mathrm{int}}^{*}}{3 \varepsilon_{\mathrm{int}}^{*}+3 C_{\mathrm{mem}}^{*} r}
$$

assuming that the thickness $d$ of the cell membrane is much less than the radius $r$ of the cell interior. The membrane capacitance $C_{\mathrm{mem}}^{*}$ is given by [28]

$$
C_{\mathrm{mem}}^{*}=\frac{\varepsilon_{\mathrm{mem}}}{d}-\frac{j \sigma_{\mathrm{mem}}}{d}
$$

A different DEP force is experienced by polystyrene beads and mammalian cells, as a result of their difference in electrical properties [Fig. 14(b)]. Typical parameters for polystyrene beads, red blood cells, and B cells were used to simulate the change in DEP response for each particle type, which was suspended in an aqueous solution with a conductivity of $10 \mathrm{mS} / \mathrm{m}$ (Table II). Empirically, this means that under the experimental conditions described in this paper, polystyrene beads will always experience a repulsive force (negative values) from the OET optical patterns. In contrast, red blood cells and B cells experience a repulsive force at lower frequencies (less than approximately $100 \mathrm{kHz}$ ) and an attractive force at higher frequencies (greater than approximately $100 \mathrm{kHz}$ ).

The concentration of bovine red blood cells was achieved using a $0.8-\mathrm{mW} \mathrm{He}-\mathrm{Ne}$ laser $(\lambda=633 \mathrm{~nm})$ as an optical

MiCROPARTICLE PROPERTIES

\begin{tabular}{llll}
\hline \hline & Polystyrene & Red blood cell & B cell $^{\mathrm{b}}$ \\
\hline$\varepsilon_{\mathrm{r}}$ & 2.55 & $\mathrm{n} / \mathrm{a}$ & $\mathrm{n} / \mathrm{a}$ \\
$\sigma$ & $0.1 \mathrm{mS} / \mathrm{m}$ & $\mathrm{n} / \mathrm{a}$ & $\mathrm{n} / \mathrm{a}$ \\
$\varepsilon_{\text {int }}$ & $\mathrm{n} / \mathrm{a}$ & 50 & 154 \\
$\sigma_{\text {int }}$ & $\mathrm{n} / \mathrm{a}$ & $0.53 \mathrm{~S} / \mathrm{m}$ & $0.73 \mathrm{~S} / \mathrm{m}$ \\
$\mathrm{C}_{\text {mem }}$ & $\mathrm{n} / \mathrm{a}$ & $0.01 \mathrm{~F} / \mathrm{m}^{2}$ & $0.0126 \mathrm{~F} / \mathrm{m}^{2}$ \\
Radius & $\mathrm{n} / \mathrm{a}$ & $2.4 \mu \mathrm{m}$ & $3.29 \mu \mathrm{m}$ \\
\hline
\end{tabular}

${ }^{\mathrm{a}}$ Red blood cell parameters are from reference [29]

${ }^{\mathrm{b}} \mathrm{B}$ cell parameters are from reference [30]

source. A spatial light modulator was not used in the setup. A $10 \times$ objective lens was used to reduce the laser beam size to $\sim 20 \mu \mathrm{m}$ in diameter.

A strong positive OET response was observed at an applied ac bias of $3 \mathrm{Vpp}$ at $200 \mathrm{kHz}$, attracting the red blood cells toward the laser spot (Fig. 15). Initially, the laser is on [Fig. 15(a)], but no electric field is applied. An ac bias is then applied to the OET device, producing OET force, which attracts the blood cells to the illuminated area at velocities of up to $9 \mu \mathrm{m} / \mathrm{s}$ [Fig. 15(b)]. It was also observed that the cells align vertically along the electric field lines [Fig. 14(b)]. When the laser is turned off, the concentrated cells remain in the area that the laser spot was focused [Fig. 15(c)]. As the applied voltage is switched off, the concentrated red blood cells began to slowly pulsate, migrating away from the central area [Fig. 15(d)], implying that they remain alive and viable.

The patterning of live human B cells was achieved with a 100-W halogen lamp and the DMD spatial light modulator. The B cells can be manipulated into an arbitrary pattern. Here, we chose to assemble the cells into the shape of " $U$ " and " $C$ " 

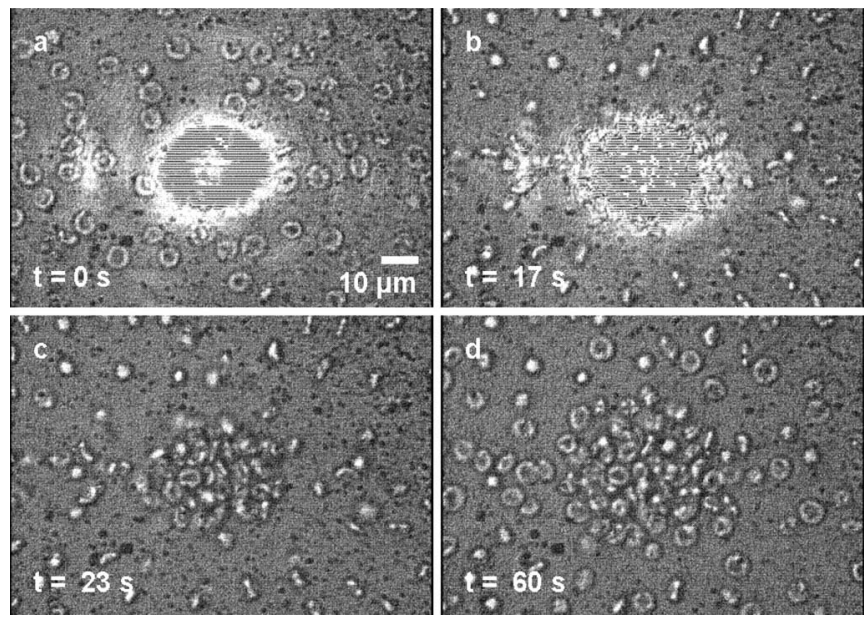

Fig. 15. Concentration of bovine red blood cells using OET. (a) Prior to applying an electric field, the blood cells are not attracted to the laser spot. (b) Applied voltage of $3 \mathrm{Vpp}$ at $200 \mathrm{kHz}$ is switched on. The red blood cells align vertically with the electric field and are attracted to the laser spot. (c) Laser and applied voltage are switched off. Red blood cells are clearly shown to be concentrated in the area of the laser spot. (d) Concentrated cells slowly diffuse out of the concentrated area.
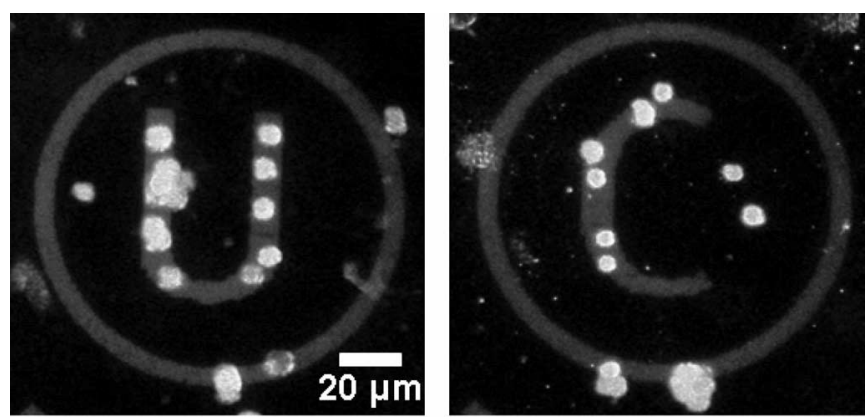

Fig. 16. Patterning of human white blood cells. Cells are attracted to the illuminated " $U$ " and " $C$ " characters by positive OET. The ring is part of a concentric pattern that is used to concentrate the B-lymphocytes toward the characters. Additional cells along the ring are moving in toward the characters.

characters (for University of California). At an applied bias of $14 \mathrm{Vpp}$ at a frequency of $100 \mathrm{kHz}$, the white blood cells exhibit positive OET behavior. A shrinking concentric ring pattern is used to concentrate the cells toward the character image at velocities of approximately $9 \mu \mathrm{m} / \mathrm{s}$ (Fig. 16). Cells are attracted to each concentric ring. As the rings shrink, the cells are transported toward the center of the concentric rings, where the character image is projected. The cells then become trapped by the static character pattern. The time required to form each character is approximately 1-2 min, depending on the overall cell concentration in the sample and the local concentration of the cells in the area covered by the optical pattern.

\section{Discussion}

OET offer a few advantages over other microparticle manipulation techniques. Conventional DEP employs static electrodes patterned with photolithographic techniques and is, thus, not reconfigurable. Furthermore, our device requires no photolithography, making fabrication easier and less expensive. Reconfigurable DEP arrays have been demonstrated using
CMOS technology [24], but these devices are complicated and expensive to fabricate. In contrast, OET devices require minimal fabrication and no photolithography. The low fabrication costs of OET make it amenable for use as a disposable device, which reduces the risk of cross-contamination of samples.

The effective manipulation area of typical optical tweezers is limited by the high-numerical-aperture objective lenses that are used to focus the laser beam. These lenses have a typical field of view of less than $100 \mu \mathrm{m}$, limiting the parallel manipulation capabilities of larger microparticles (e.g., human lymphocytes have diameters of $\sim 10 \mu \mathrm{m})$. Since the OET mechanism does not require a highly focused beam, low-magnification objectives allow OET to be effective over a much larger area $(1.3 \times 1.0 \mathrm{~mm})$. We have demonstrated this by the creation of an array of 15000 individually addressable single-particle traps over an area of $1.3 \mathrm{~mm}^{2}$ [15]. The array consists of optically defined traps with diameters of $4.5 \mu \mathrm{m}$, containing 4.5- $\mu \mathrm{m}$-diameter polystyrene beads, with a trap-to-trap spacing of $9 \mu \mathrm{m}$. However, a tradeoff exists between OET resolution and effective manipulation area; to create a more highly focused optical pattern, the effective area must be reduced. This optical pattern limitation does not apply to electrode-based DEP, where the effective manipulation area is limited only by constraints on device dimensions. However, the reconfigurable nature of OET can be used to mitigate the effects of a limited manipulation area. For example, conventional DEP sorts similarly sized particles based solely on differences in their electrical properties [21], [23], [28]. With OET, we can use visual attributes such as size, color, and texture to further differentiate between particles. Once distinguishing visual attributes have been identified using image-analysis software, the appropriate optical patterns can be generated to separate the different particles [27]. With this system, it is possible to separate particles over a smaller area than would be required otherwise.

Although holographic tweezers can generate multiple dynamic traps, direct imaging using a DMD is more versatile. Any arbitrary pattern can be generated with a high contrast ratio, without any of the computation associated with the generation of a holographic pattern. Furthermore, OET can induce repulsive forces on transparent dielectric particles such as biological cells, whereas optical tweezers is limited to attractive forces.

As OET relies on a different mechanism than optical tweezers, the optical power requirements of OET are much lower than those of optical tweezers. A typical optical tweezers trap requires at least $1 \mathrm{~mW}$ of optical power, whereas the creation of multiple optical traps with holographic optical tweezers requires lasers with powers of $500 \mathrm{~mW}$ to $1 \mathrm{~W}$ [5], [6]. In contrast, OET actuation has been obtained using power levels as low as $1 \mu \mathrm{W}$, corresponding to an optical power density of $4.4 \mathrm{nW} / \mu \mathrm{m}^{2}$ [16]. This is five orders of magnitude lower than the power density in a $1-\mathrm{mW}$ optical tweezers trap that is focused at the diffraction limit. The much lower power requirements of OET actuation allow the use of inexpensive incoherent light sources. In addition, the lower power densities in OET traps lower the risk of optical damage or heating effects on live biological particles [7], [8]. The optical power density has an effect on the force induced in the OET device, as higher 
optical intensities further reduce the impedance of the a-Si layer. In the experiments described here, the incoherent light sources do not generate sufficient optical intensities to fully turn on the virtual electrode that is created by the optical pattern. As a result, the OET forces are lower than in previous results [16] and similar electrode-based DEP devices. Stronger OET forces may be obtained by increasing the intensity of the optical source or by improving the photoresponse of the a-Si layer.

The photoconductivity of the a-Si layer also limits the conductivity of the liquid layer. As described earlier, OET relies on the switching of the applied voltage between the liquid and a-Si layers of the OET device. Thus, the impedance of the illuminated a-Si must be less than that of the liquid layer. While this condition is met by low-conductivity media such as those used in the experiments described here, high-conductivity media such as cell culture media is incompatible with OET operation. Our group is currently working on improving the OET device for use with high-conductivity liquids.

We envision the OET device as a tool to complement conventional DEP and optical tweezers. The optically patterned electrodes of OET allow greater flexibility than those of conventional DEP, such as the ability to isolate specific single particles from a mixed population, which could be useful in applications such as stem-cell research. In addition, the optical requirements for OET are more relaxed than those for optical tweezers, offering greater flexibility in optical sources and reducing the risk of photodamage to the particles under manipulation. The flexibility and dynamic control of OET manipulation will provide a powerful tool in biological experiments.

\section{CONCLUSiON}

We have demonstrated optical particle manipulation using incoherent light. By integrating a spatial light modulator and direct imaging with OET, many dynamic reconfigurable manipulation patterns can be employed. Using a variety of patterns, we have realized live cell and microparticle collectors, singleparticle traps, and individually addressable single-particle arrays. In addition, we have achieved other manipulation functions unique to OET-based manipulation, such as light-defined particle channels and size-based particle sorting with a comb sorter. Such particle manipulation techniques have many applications to experiments with biological cells and microparticles.

\section{REFERENCES}

[1] A. Ashkin, J. M. Dziedzic, J. E. Bjorkholm, and S. Chu, "Observation of a single-beam gradient force optical trap for dielectric particles," Opt. Lett., vol. 11 , no. 5, pp. 288-290, May 1986.

[2] M. P. MacDonald, G. C. Spalding, and K. Dholakia, "Microfluidic sorting in an optical lattice," Nature, vol. 426, no. 6965, pp. 421-424, Nov. 2003.

[3] M. P. MacDonald, L. Paterson, K. Volke-Sepulveda, J. Arlt, W. Sibbett, and K. Dholakia, "Creation and manipulation of three-dimensional optically trapped structures," Science, vol. 296, no. 5570, pp. 1101-1103, May 2002.

[4] D. G. Grier, "A revolution in optical manipulation," Nature, vol. 424, no. 6950, pp. 810-816, Aug. 2003.

[5] W. J. Hossack, E. Theofanidou, J. Crain, K. Heggarty, and M. Birch, "High-speed holographic optical tweezers using a ferroelectric liquid crystal microdisplay," Opt. Express, vol. 11, no. 17, p. 2053, Aug. 2003.

[6] J. E. Curtis, B. A. Koss, and D. G. Grier, "Dynamic holographic optical tweezers," Opt. Commun., vol. 207, no. 1, pp. 169-175, Jun. 2002.
[7] A. Ashkin and J. M. Dziedzic, "Optical trapping and manipulation of viruses and bacteria," Science, vol. 235, no. 4795, pp. 1517-1520, Mar. 1987.

[8] A. Ashkin, J. M. Dziedzic, and T. Yamane, "Optical trapping and manipulation of single cells using infrared laser beams," Nature, vol. 330, no. 6150, pp. 769-771, Dec. 1987.

[9] Y. Liu, G. J. Sonek, M. W. Berns, and B. J. Tromberg, "Physiological monitoring of optically trapped cells: Assessing the effects of confinement by 1064-nm laser tweezers using microfluorometry," Biophys. J., vol. 71, no. 4, pp. 2158-2167, Oct. 1996.

[10] H. Liang, K. T. Vu, P. Krishnan, T. C. Trang, D. Shin, S. Kimel, and M. W. Berns, "Wavelength dependence of cell cloning efficiency after optical trapping," Biophys. J., vol. 70, no. 3, pp. 1529-1533, Mar. 1996.

[11] K. C. Neuman, E. H. Chadd, G. F. Liou, K. Bergman, and S. M. Block, "Characterization of photodamage to Escherichia coli in optical traps," Biophys. J., vol. 77, no. 5, pp. 2856-2863, Nov. 1999.

[12] S. K. Mohanty, A. Rapp, S. Monajembashi, P. K. Gupta, and K. O. Greulich, "Comet assay measurements of DNA damage in cells by laser microbeams and trapping beams with wavelengths spanning a range of $308 \mathrm{~nm}$ to $1064 \mathrm{~nm}$," Radiat. Res., vol. 157, no. 4, pp. 378-385, 2002.

[13] H. A. Pohl, Dielectrophoresis. Cambridge, U.K.: Cambridge Univ. Press, 1978.

[14] T. Muller, A. Gerardino, T. Schnelle, S. G. Shirley, F. Bordoni, G. De Gasperis, R. Leoni, and G. Fuhr, "Trapping of micrometre and submicrometre particles by high-frequency electric fields and hydrodynamic forces," J. Phys. D, Appl. Phys., vol. 29, no. 2, pp. 340-349, 1996.

[15] P. Y. Chiou, A. T. Ohta, and M. C. Wu, "Massively parallel manipulation of single cells and microparticles using optical images," Nature, vol. 436, no. 7049, pp. 370-372, Jul. 2005.

[16] P. Y. Chiou, Z. Chang, and M. C. Wu, "A novel optoelectronic tweezer using light induced dielectrophoresis," in Proc. IEEE/LEOS Int. Conf. Opt. MEMS, Kona, HI, 2003, pp. 8-9.

[17] P. Y. Chiou, W. Wong, J. C. Liao, and M. C. Wu, "Cell addressing and trapping using novel optoelectronic tweezers," in Proc. 17th IEEE Int. Conf. MEMS, Maastricht, The Netherlands, 2004, pp. 21-24.

[18] Y.-S. Lu, Y.-H. Chang, S.-P. Tseng, and J. A. Yeh, "Virtual particle channels based on optical dielectrophoresis forces," in Proc. IEEE/LEOS Int. Conf. Opt. MEMS, Takamatsu, Japan, 2004, pp. 20-21.

[19] Y.-S. Lu, Y.-P. Huang, J. A. Yeh, and C. Lee, "Image driven cell manipulation using optical dielectrophoresis (ODEP)," in Proc. 2nd Asian and Pacific Rim Symp. Biophotonics, Taipei, Taiwan, R.O.C., 2004, pp. 238-239.

[20] Y.-S. Lu, Y.-P. Huang, J. A. Yeh, C. Lee, and Y.-H. Chang, "Controllability of non-contact cell manipulation by image dielectrophoresis (iDEP)," Opt. Quantum Electron., vol. 37, no. 13-15, pp. 1385-1395, Dec. 2005.

[21] P. R. C. Gascoyne and J. V. Vykoukal, "Dielectrophoresis-based sample handling in general-purpose programmable diagnostic instruments," Proc. IEEE, vol. 92, no. 1, pp. 22-42, Jan. 2004.

[22] H. Morgan, M. P. Hughes, and N. G. Green, "Separation of submicron bioparticles by dielectrophoresis," Biophys. J., vol. 77, no. 1, pp. 516-525, Jul. 1999.

[23] R. Pethig, M. S. Talary, and R. S. Lee, "Enhancing traveling-wave dielectrophoresis with signal superposition," IEEE Eng. Med. Biol. Mag., vol. 22, no. 6, pp. 43-50, Nov./Dec. 2003.

[24] N. Manaresi, A. Romani, G. Medoro, L. Altomare, A. Leonardi, M. Tartagni, and R. Guerrieri, "A CMOS chip for individual cell manipulation and detection," IEEE J. Solid-State Circuits, vol. 38, no. 12, pp. 2297-2305, Dec. 2003.

[25] COMSOL Multiphysics Finite-Element Modeling Software, COMSOL, Inc., Burlington, MA. [Online]. Available: http://www.comsol.com/ products/multiphysics/

[26] P. Y. Chiou, A. T. Ohta, and M. C. Wu, "Microvision-activated automatic optical manipulator for microscopic particles," in Proc. 18th IEEE Int. Conf. MEMS, Miami, FL, 2005, pp. 682-685.

[27] P. Y. Chiou, A. T. Ohta, and M. C. Wu, "Continuous optical sorting of HeLa cells and microparticles using optoelectronic tweezers," in Proc. IEEE/LEOS Int. Conf. Opt. MEMS, 2005, pp. 83-84.

[28] P. R. C. Gascoyne, X. B. Wang, Y. Huang, and F. F. Becker, "Dielectrophoretic separation of cancer cells from blood," IEEE Trans. Ind. Appl., vol. 33, no. 3, pp. 670-678, May/Jun. 1997.

[29] J. Gimsa and D. Wachner, "A unified resistor-capacitor model for impedance, dielectrophoresis, electrorotation, and induced transmembrane potential," Biophys. J., vol. 75, no. 2, pp. 1107-1116, Aug. 1998.

[30] J. Yang, Y. Huang, X. J. Wang, X. B. Wang, F. F. Becker, and P. R. C. Gascoyne, "Dielectric properties of human leukocyte subpopulations determined by electrorotation as a cell separation criterion," Biophys. J., vol. 76, no. 6, pp. 3307-3314, Jun. 1999. 


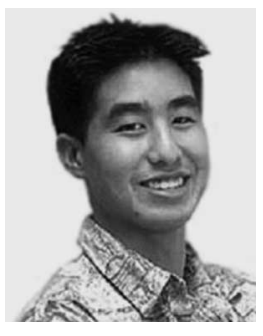

Aaron Takami Ohta (S'99) received the B.S. degree from the University of Hawaii, Manoa, in 2003, and the M.S. degree from the University of California, Los Angeles, in 2004, both in electrical engineering. $\mathrm{He}$ is currently working toward the Ph.D. degree in electrical engineering at the University of California, Berkeley.

His research interests include MEMS and bioMEMS.

Mr. Ohta was a recipient of a National Science Foundation Graduate Research Fellowship.

Pei-Yu Chiou received the B.S. degree in mechanical engineering from National Taiwan University, Taipei, Taiwan, R.O.C., the M.S. degree in electrical engineering from the University of California, Los Angeles (UCLA), and the Ph.D. degree in electrical engineering and computer sciences from the University of California, Berkeley (UC Berkeley), in 2005.

He joined the faculty of UCLA as a member of the Department of Mechanical and Aerospace Engineering in 2006, after postdoctoral work at UC Berkeley. His research interests are in bioMEMS and biophotonics.

Tae H. Han, photograph and biography not available at the time of publication.

James C. Liao received the B.S. degree in chemical engineering from National Taiwan University, Taipei, Taiwan, R.O.C., and the Ph.D. degree from the University of Wisconsin, Madison, in 1987.

After working as a Research Scientist in the Life Science Research Laboratory, Eastman Kodak Company, Rochester, NY, he started his academic career at Texas A\&M University, College Station, in 1990, as an Assistant Professor and then an Associate Professor. He moved to the University of California, Los Angeles, in 1997, as a Full Professor and is currently serving as the Vice Chair of the Department of Chemical and Biomolecular Engineering. $\mathrm{He}$ is a pioneer in the field of metabolic engineering, synthetic biology, and systems biology. His development of mathematical and genetic analyses of gene-metabolic networks has made significant impact in both fundamental research and industrial applications.

Urvashi Bhardwaj, photograph and biography not available at the time of publication.

Edward R. B. McCabe received the B.S. degree from The Johns Hopkins University, Baltimore, MD, in 1967, and the Ph.D. and M.D. degrees from the University of Southern California, Los Angles, in 1972 and 1974, respectively.

He completed a Pediatric Residency (1974-1976) at the University of Minnesota, Minneapolis, and a Metabolism Fellowship (1976-1978) at the University of Colorado Health Science Center (UCHSC), Denver. As a Fellow, he discovered glycerol kinase deficiency and proceeded to characterize the biochemical nature of this disorder while he was an Instructor (1977-1978), Assistant Professor (1978-1982), and Associate Professor (1982-1986) at UCHSC. In 1986, he moved to Baylor College of Medicine, Houston, TX, as an Associate Professor (1986-1988) and Professor (1988-1994). At Baylor, he cloned the gene involved in glycerol kinase deficiency and the gene for adrenal hypoplasia congenita. He moved to the University of California, Los Angeles, as a Professor and the Executive Chair of the Department of Pediatrics (1994-present) and joined the Department of Human Genetics (2000-present). Noting a lack of genotype/phenotype correlation for these single-gene disorders, his research focuses on them as complex traits and explores the systems biology of each disorder.
Fuqu Yu is a graduate student in molecular and medical pharmacology at the University of California, Los Angeles.

Ren Sun received the Ph.D. degree from Yale University School of Medicine, New Haven, CT.

He obtained his postdoctoral training in the Department of Molecular Biophysics and Biochemistry, Yale University School of Medicine. He joined the University of California, Los Angeles, in 1998, as an Assistant Professor in the Department of Molecular and Medical Pharmacology and became an Associate Professor in 2003. He studied tumor-associated herpesviruses, EpsteinBarr virus, Kaposi's sarcoma-associated herpesvirus (KSHV), and murine herpesvirus-68. He identified the KSHV-encoded polyadenylated nuclear RNA, which represents a new type of RNA, possessing features of both U snRNA and mRNA. He also identified the replication and transcription activator as the master switch protein that disrupts latency and initiates the lytic cycle.

Dr. Sun is a member of the Molecular Biology Institute, the AIDS Institute, the Jonsson Comprehensive Cancer Center, the Dental Research Institute, and the California NanoSystems Institute.

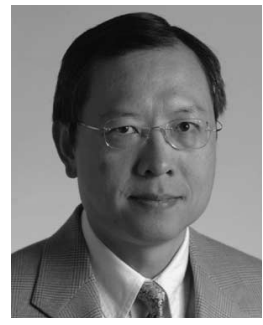

Ming C. Wu (S'82-M'83-SM'00-F'02) received the B.S. degree in electrical engineering from $\mathrm{Na}-$ tional Taiwan University, Taipei, Taiwan, R.O.C., and the M.S. and Ph.D. degrees in electrical engineering and computer sciences from the University of California, Berkeley, in 1985 and 1988, respectively.

From 1988 to 1992, he was a Member of Technical Staff at AT\&T Bell Laboratories, Murray Hill, NJ. From 1992 to 2004, he was a Professor in the Department of Electrical Engineering, University of California, Los Angeles, where he also served as the Vice Chair for the Industrial Affiliate Program and the Director of the Nanoelectronics Research Facility. In 2004, he moved to the University of California, Berkeley, where he is currently a Professor of electrical engineering and computer sciences. He is also a Co-Director of the Berkeley Sensor and Actuator Center. He is the author or coauthor of six book chapters, more than 135 journal papers, and 280 papers published in conference proceedings. He is the holder of 14 U.S. patents. His research interests include optical microelectromechanical systems, high-speed semiconductor optoelectronics, nanophotonics, and biophotonics.

Prof. Wu is a member of the Optical Society of America. He was a Packard Foundation Fellow from 1992 to 1997. He is the founding Co-Chair of the IEEE/LEOS Summer Topical Meeting on Optical MEMS (1996) and the predecessor of the IEEE/LEOS International Conference on Optical MEMS. $\mathrm{He}$ has served on the program committees of many technical conferences, including MEMS, OFC, CLEO, LEOS, MWP, IEDM, DRC, and ISSCC. He has also served as a Guest Editor of two special issues of IEEE journals on Optical MEMS. 